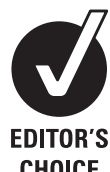

CHOICE

${ }^{1}$ Université Joseph Fourier, Grenoble I, Grenoble, France ${ }^{2}$ INSERM, Grenoble Institut des Neurosciences,

INSERM-UJF-CEA-CHU U836, Grenoble, France

${ }^{3}$ Centre Hospitalier Universitaire (CHU), Service de Neurologie, Grenoble, France

${ }^{4}$ Hôpitaux Universitaires de Genève, Genève, Switzerland

\section{Correspondence to}

A Maillet, Grenoble Institut des Neurosciences, U836 Inserm-UJF-CEA-CHU, Pavillon de Neurologie, Centre Hospitalier Universitaire de Grenoble, BP 217, 38043 Grenoble Cedex 9, France; amaillet@chu-grenoble.fr

Received 7 February 2012 Revised 22 May 2012

Accepted 23 May 2012 Published Online First 8 July 2012

REVIEW

\title{
Imaging gait disorders in parkinsonism: a review
}

\author{
Audrey Maillet, ${ }^{1,2}$ Pierre Pollak, ${ }^{1,2,3,4}$ Bettina Debû ${ }^{1,2}$
}

\section{ABSTRACT}

Gait difficulties-including freezing of gait-are frequent and disabling symptoms of advanced Parkinson's disease and other parkinsonian syndromes. They respond poorly to current medical and surgical treatments, making patient management very difficult. The underlying pathophysiology remains largely unknown. The late onset of levodopa resistance of Parkinson's disease gait abnormalities has been suggested to result from the progressive extension of the degenerative process to non-dopaminergic structures involved in locomotion, such as cortico-frontal and brainstem networks. Deficiencies in other neurotransmission systems, involving acetylcholine, serotonin or norepinephrine, have also been evoked. Neuroimaging tools appear well suited to decipher the cerebral substrates of parkinsonian gait disorders and their modulation by dopaminergic medication or deep brain stimulation. Here the main functional and metabolic neuroimaging studies aimed at identifying these cerebral networks are reviewed, in both healthy subjects and parkinsonian patients. After a brief overview of the physiology and pathophysiology of gait control, the methodology, main results and limits of the studies published to date are examined. The most promising methods to examine gait difficulties and their response to currently available treatments are then discussed.

\section{INTRODUCTION}

Gait disorders are frequent and disabling symptoms of advanced Parkinson's disease (PD). ${ }^{1}$ In addition to the typical small steps, shuffling gait of $\mathrm{PD}$, some patients experience freezing of gait (FoG), a sudden and unexpected inability to start or continue walking that can be responsible for falls. Gait impairments are also seen in atypical parkinsonism, a heterogeneous spectrum of pathologies, including progressive primary freezing of gait, progressive supranuclear palsy (PSP), multiple system atrophy, Lewy body disease, cortico-basal degeneration (CBD), normal pressure hydrocephalus and vascular parkinsonism. ${ }^{2-4}$ These diseases are marked by the early onset of gait and balance disorders, isolated FoG or 'pure' akinesia with gait freezing.

Gait difficulties greatly impact on mobility, leading to a progressive loss of autonomy and a decrease in the patient's quality of life. ${ }^{1}$ In $\mathrm{PD}$, gait difficulties are initially improved by dopaminergic medication, or subthalamic nucleus (STN) or internal globus pallidus stimulation, but become progressively resistant to these therapies in patients with advanced disease. ${ }^{5}$ In addition, it has recently been shown that pedunculopontine nucleus (PPN) deep brain stimulation (DBS) may reduce FoG and falls related to FoG in some, but not all, patients with PD. In other parkinsonian syndromes, gait disorders have a poor prognosis, being resistant to levodopa from the onset of the disease. Because the pathogenesis of gait disorders and FoG remains poorly understood, clinicians are at a loss to try and alleviate the discomfort of patients.

Although animal models have shed some light on the subcortical and brainstem structures involved in the control of locomotion, little is known of the precise involvement of these structures in humans, or their disruption in patients with gait disorders Furthermore, modulation of the underlying neural circuits by standard antiparkinsonian medication or surgical treatments is not fully understood. A better understanding of the neural circuits underlying normal gait as well as gait disorders and FoG in PD and other forms of parkinsonism is required to improve clinical care.

Functional brain imaging techniques appear ideally suited to answer these questions. However, the techniques offering the best spatial and temporal resolutions require that the head of the patient remains immobile, thus precluding actual gait execution. Mental motor imagery offers a promising alternative, provided that specific methodological precautions are taken. Here we review the neuroimaging studies aimed at deciphering the neural networks underlying gait in both healthy and parkinsonian individuals. We discuss the limitations of each technique and stress the methodological precautions that need to be taken in order to ensure reliable data.

\section{HUMAN GAIT CONTROL}

Gait depends on both automatic and volitional processes. Supraspinal structures, such as the mesencephalic locomotor region (MLR) and the pontomedullary reticular formation, activate and regulate spinal pattern generators controlling the basic step cycle. ${ }^{6}$ In addition, functional and efficient gait (navigation through space, obstacle avoidance or adaptation to the goal) depends on higher level control centres, including the basal ganglia (BG), cerebellum, and parietal and frontal cortical areas. ${ }^{7}$

Gait disturbances are a hallmark of parkinsonian syndromes and can take different forms. In addition to the typical parkinsonian gait, some patients may experience festination and FoG. FoG is a sudden and unexpected inability to walk. ${ }^{1}{ }^{2}$ Festination is an involuntary shortening and fastening of gait. ${ }^{1}$ Both symptoms are influenced by factors such as the environment, task demand, medication, attention and emotions. ${ }^{1}$

The pathophysiology of these disorders remains poorly understood. In $\mathrm{PD}$, the late onset levodopa resistance of the symptom suggests the 
involvement of cerebral networks different from the ones responsible for akinesia. Specifically, the hypothesis of a progressive extension of the degenerative process to nondopaminergic structures controlling locomotion has been put forward. ${ }^{8}$ Cortico-frontal and brainstem lesions have been proposed, as well as disruption in the circuits connecting the BG and MLR, including the PPN. ${ }^{9}$ In atypical parkinsonism, the association of nigrostriatal dopaminergic loss with either cortical lesions, in the case of CBD and Lewy body disease, and/or brainstem lesions, in the case of PSP, could explain both the severity of the symptoms and the lack of significant improvement following dopaminergic therapy.

Regarding FoG, its occurrence in disorders other than PD also suggests that deficiencies in neurotransmitters other than dopamine could be involved. This idea is supported by the variable response of FoG to dopaminergic drugs. Defects in glutamate, norepinephrine ${ }^{10}$ and serotonin have been evoked. Thus early onset and severe (up to 70\%) norepinephrine depletion occurs in the locus coeruleus and has been suggested to significantly contribute to motor dysfunction in PD. ${ }^{11}$ FoG could also result from abnormal norepinephrine release in the frontal lobe. ${ }^{13}$ Degeneration of the serotoninergic neurons in the raphe nuclei is less severe. ${ }^{13}$ Finally, the role of cholinergic structures, particularly the PPN, in gait disorders and FoG has attracted a lot of attention in recent years.

The PPN has reciprocal connections with cortical and subcortical structures, such as the cerebral cortex, limbic system, BG and cerebellum, as well as descending projections to the lower brainstem and spinal cord. ${ }^{12}$ Animal studies have shown that low frequency PPN stimulation $(20-60 \mathrm{~Hz})$ can elicit locomotion in decerebrated cats while its pharmacological activation improves locomotor activity in MPTP treated monkeys. ${ }^{14}$ In contrast, lesion, pharmacological inhibition ${ }^{14}$ or high frequency stimulation (over $60 \mathrm{~Hz}$ ) of the PPN causes severe akinesia and postural disorders in monkeys. ${ }^{15}$ Finally, specific lesions of the cholinergic PPN cells induce postural abnormalities. ${ }^{15}$ In humans, gait disorders following vascular midbrain lesions or bilateral stroke ${ }^{16}$ in the PPN area (PPNa) also suggest involvement of this region in locomotion. In addition, post mortem studies have revealed that degeneration of the PPN correlates with the severity of gait difficulties before death. ${ }^{15} 17$ Loss of PPN neurons is even more important in PSP. ${ }^{17}$ Regarding anatomy, a link has been established between the diameter of the midbrain and the severity of gait disorders in patients with $\mathrm{PD}^{18}$ or chronic hydrocephaly. ${ }^{19}$

These findings lead to testing the efficacy of low frequency stimulation of the $\mathrm{PPNa}$ to alleviate gait disorders in PD. Although early reports described dramatic improvements in gait disorders and FoG, ${ }^{20}$ better controlled studies revealed a much more mitigated outcome. Improvement of FoG and decrease in falls related to FoG appear highly variable, ranging from a significant reduction in some patients to a lack of effect in others. ${ }^{22} 23$ Nevertheless, this is the first time stimulation frequencies as low as $20 \mathrm{~Hz}$ have been shown to have an effect in movement disorders. Because the relevance of stimulating a degenerating and atrophic mesencephalic structure can be questioned, a better understanding of the mechanisms underlying the influence of PPNa stimulation on gait difficulties is directly needed.

Cerebral imaging appears to be a suitable tool to study the pathophysiological mechanisms underlying gait disorders in advanced PD and parkinsonian syndromes, as well as their modulation by levodopa or PPNa stimulation. Below we review the main neuroimaging studies aimed at identifying the cerebral networks involved in gait control, in both healthy subjects and parkinsonian patients. We also discuss studies exploring modulation of these networks by medical treatment or brain surgery. We describe the methods used in these studies and their limits, and then examine the main results obtained using different locomotion or locomotion-like tasks.

\section{WHICH TOOLS TO STUDY THE CEREBRAL NETWORKS INVOLVED IN HUMAN GAIT?}

The limits of our understanding of the mechanisms underlying human gait control are mainly due to the methodological and technical constraints of the movement itself. The first difficulty stems from the lack of relevant animal models for studying bipedal gait and gait disorders. Nevertheless, data in rodents, cats and primates have revealed the involvement of cortical, subcortical and brainstem structures in gait regulation. In addition, clinical data relating lesions of the MLR and gait disorders ${ }^{16}$ strongly suggest its implication in human gait control, although they do not reveal the underlying mechanisms. In this context, neuroimaging studies fill an important gap.

Electroencephalography (EEG) and magnetoencephalography (MEG) measure cerebral activity with good temporal resolution. EEG precisely records rhythmic electrical signals and provides data regarding possible functional deteriorations of their dynamics. However, although it can be used to measure the cerebral electric activity preceding gait initiation in the upright position, ${ }^{24}$ recording the signal during walking is restricted by movement artefacts. In addition, EEG monitors the activity of neuronal ensembles located at a distance from the electrodes. While high density EEG partly resolves these limits, ${ }^{25}$ the spatial resolution of the signal is not as good as that of positron emission tomography (PET) or functional MRI (fMRI). Therefore, EEG is often used together with FMRI or PET. MEG consists of measuring the magnetic fields produced by neuronal electric activity. However, similar to EEG, MEG measures the signal generated by a number of synchronously activated neurons, which somewhat limits its spatial resolution. In addition, the technique requires the subject to sit still, precluding the performance of whole body movements. To our knowledge, no MEG study to date has been carried out on gait disorders.

Single photon emission CT (SPECT), near infrared spectroscopy (NIRS), fMRI and PET enable the study of neurovascular coupling-that is, the link between increased neuronal activity and increased metabolism occurring during performance of a specific task. Specifically, SPECT and PET monitor the disintegration kinetics of a radiotracer-such as ${ }^{18} \mathrm{FDG}$ or $\mathrm{H}_{2} 0^{15}$ for PET, ${ }^{99 m}[\mathrm{Tc}]-\mathrm{HM}-\mathrm{PAO}$ or ${ }^{123}$ [I]-IMP for SPECT $\rightarrow$ while fMRI measures the BOLD (blood oxygenation level dependent) signal variations associated with changes in the blood oxygen concentration downstream of the activated neurons. NIRS detects small changes in haemoglobin oxygenation, and its physical principles are similar to those of fMRI. NIRS can be used in ambulatory mode and thus can be used to study actual gait performance. However, the infrared light can only penetrate a few centimetres below the skull surface, making it impossible to acquire data from deep brain structures. In addition, both spatial and temporal resolutions are weak.

The half-life duration of the radiotracers used in SPECT is long. Once injected, they are quickly distributed and are retained in the brain for several hours. This allows for execution of a specific task, such as freely moving gait, during which the radiotracer is fixed in the active brain regions, followed by image acquisition. The whole brain, including the BG and cerebellum, can be scanned using SPECT. Thus SPECT could prove useful to differentiate the neural substrates involved in the different types 
of gait disorders in parkinsonism (shuffling, akinetic or trembling FoG, instability) in well characterised groups of patients. However, if SPECT enables the description of the cerebral structures involved in gait maintenance, it does not shed light on gait initiation, as subjects must walk before and after tracer injection. SPECT also limits the study to a few experimental conditions because of the long half-life of the radiotracers. Finally, the spatial resolution of SPECT is also somewhat limited.

PET and FMRI have the best spatial resolution and enable image acquisition of deep brain structures as well as cortical areas. Their spatial resolution is better than that of SPECT, enabling the comparison of several conditions or tasks within a single experimental session. However, fMRI use in patients carrying intracerebral electrodes raises safety issues. Thus PET or SPECT are usually used in patients with DBS. Finally, using specific ligands and well characterised groups of patients, PET and SPECT could also be useful to study the neurotransmitter alterations associated with particular gait symptoms. However, both techniques involve radiation exposure.

Although FMRI and PET are the two favoured techniques for studying the cerebral network involved in voluntary movements, obtaining good quality images requires that the head of the participant does not move. Therefore, these techniques mainly allow the study of small amplitude upper arm movements or repetitive leg or foot movements ${ }^{26} 27$ while lying or seating, excluding the actual execution of gait. This constraint is likely responsible for the relative scarcity of cerebral imaging studies of locomotion. As no portable system enabling the real time recording of cerebral activity during locomotion currently exists, alternative protocols have been developed.

\section{FROM METABOLIC STUDIES TO FUNCTIONAL NEUROIMAGING OF NORMAL AND PARKINSONIAN GAIT}

Metabolic studies in PD and parkinsonian syndromes

Following the idea that PD alterations are also present in the cerebral activity at rest, some authors used SPECT or PET to investigate abnormalities of baseline cerebral perfusion in the resting state $e^{28-31}$ in $\mathrm{PD}$ patients with or without gait disturbances. Comparing patients with FoG with patients without FoG or healthy subjects, these studies highlighted hypometabolism in the orbitofrontal and parietal cortices, ${ }^{28-30}$ as well as in the $\mathrm{BG}^{30}$ in patients with FoG. Another study reported hypoperfusion within the right anterior cingulate cortex (CCA) and primary visual cortex in patients with postural instability and gait difficulty, compared with tremor dominant PD patients and controls. ${ }^{31}$ In addition, reduction of thalamic cholinergic markers, possibly reflecting loss of cholinergic projections from the PPN, appears related to the propensity to fall in PD patients. ${ }^{32}$

Regarding atypical parkinsonism, hypermetabolism in the frontal cortex with hypometabolism in both the BG and cerebellum have been reported in multiple system atrophy, ${ }^{33} 34$ while supplementary motor area (SMA), anterior cingulate, thalamic and BG hypometabolism are seen in PSP. ${ }^{28} 3435$ Other data show hypometabolism in the BG and midbrain in 'pure' akinesia with gait freezing compared with controls. ${ }^{35}$ Finally, asymmetric fronto-parietal hypoactivation is seen in CBD. ${ }^{34}$

A few authors also investigated brain abnormalities at rest in PD patients, after performance of an actual gait task. ${ }^{36} 37$ They reported hypometabolism in the orbitofrontal cortex and caudate nucleus in these patients, suggesting that a reduction in striatal dopamine transporters could be related to gait impairment. ${ }^{36}$ Another study compared regional cerebral blood flow
$(\mathrm{rCBF})$ changes before and after a gait rehabilitation programme in PD. A beneficial effect of the therapy was observed both on physical performance and supraspinal locomotor control, as rehabilitation somewhat reversed the hypometabolism of the right parietal and temporal cortices observed before therapy. ${ }^{37}$

Overall, these results are consistent and suggest specific alterations of resting brain activity in patients with gait difficulties. However, they do not provide information regarding the specific cerebral dysfunctions related to gait disorders in parkinsonian patients.

\section{Repetitive foot or leg movements}

Some studies used repetitive lower limb movements in the supine position as a surrogate of gait. The rationale is that, similar to gait, reciprocal foot flexion-extension or bicycle movements require internal pacing and interlimb coordination. Indeed, in healthy subjects, alternating foot movements and walking on a treadmill activate similar cortical areas differing from those related to upper limb movements. Other studies confirmed activations within the somatosensory cortices, SMA and cerebellum. ${ }^{27}$ These results thus suggest that both cortical and subcortical areas are involved in the motor control of rhythmic lower limb movements. However, repetitive foot or lower limb movement performed while lying supine lack several important features of gait control.

\section{Actual gait}

In healthy subjects, NIRS studies revealed activations in the prefrontal, ${ }^{263839}$ premotor and sensorimotor ${ }^{2638}$ cortices during walking on a treadmill. Holtzer et $a l^{39}$ found that prefrontal activation was reduced in older subjects, compared with younger ones, in relation to decreased attentional resources, executive dysfunction and gait disorders. SPECT data revealed increased activation of the SMA, premotor and visual cortices, BG and cerebellum in healthy subjects. ${ }^{40}$ In contrast, $\mathrm{PD}$ patients without gait disorders showed fronto-parietal, BG and cerebellar hemisphere hypoactivation, as well as hyperactivation within the CCA, temporal cortex and cerebellar vermis. ${ }^{41}$ In both groups, there was also increased rCBF in the dorsal brainstem. Visual cues during gait on a treadmill induced activations in the premotor, parietal cortices and cerebellar hemispheres in $\mathrm{PD}$ patients with FoG compared with controls, suggesting recruitment of cerebral areas involved in visuomotor control to overcome FoG. ${ }^{42}$

Only one SPECT study examined patients with gait difficulties attributed to atypical vascular parkinsonism during walking on a treadmill. Results revealed underactivation in the SMA, thalamus and BG, and overactivation of the premotor cortex, compared with patients without gait difficulties. ${ }^{43}$

\section{Effects of deep brain stimulation at rest or during lower limb movement in PD}

A few studies examined the effect of $\mathrm{STN}^{44}$ or $\mathrm{PPNa}^{45-47}$ stimulation on $\mathrm{rCBF}$ using $\mathrm{H}_{2} 0^{15}$-PET in $\mathrm{PD}$ patients with gait disorders. Lyoo et $\mathrm{al}^{44}$ showed that improvement in the Unified Parkinson's Disease Rating Scale motor score following STN stimulation was correlated with increased metabolism activity in the prefrontal cortex, SMA and CCA at rest, whereas FoG improvement was associated with metabolic activity in parietal, occipital and temporal sensory association cortices. Studies evaluating the effect of unilateral PPNa stimulation at rest ${ }^{45}$ or during self-paced alternating lower limb movements ${ }^{46}$ showed a bilateral increase in rCBF in the thalamus, putamen, cerebellum and MLR, correlated with $\mathrm{rCBF}$ changes in the 
sensorimotor cortex and SMA. This was the first evidence of a PPN induced rCBF modulation in both cortical and subcortical areas.

Thus, overall, there is good agreement in the literature regarding cortical activation associated with the control of normal and parkinsonian gait. However, many questions remain regarding subcortical and brainstem activation, as well as the relation between these areas and other cortical and subcortical structures involved in motor control. Moreover, little is known about the influence of treatments. Some of these issues can be addressed using a different methodological approach-namely, motor imagery.

\section{MOTOR IMAGERY: A NEW WAY TO STUDY THE CEREBRAL NETWORKS OF GAIT?}

In the past few years, a number of studies have used mental motor imagery (MI) to better understand the neuronal basis of normal or pathological locomotion. MI has long been used for training motor skills in sports ${ }^{48}$ and or relearning motor skills in rehabilitation settings. ${ }^{48}$ In addition, MI allows investigation of the internal dynamics of movement planning and preparation, while avoiding sensory and motor compounds related to motor execution. ${ }^{49-51}$ Thus, associated with brain imaging, MI enables the exploration of the cerebral networks involved in upper limb movements ${ }^{52}$ as well as in gait control. Furthermore, it has been shown that the cerebral plasticity occurring during skill acquisition could be seen both during actual movement and MI.

\section{What is motor imagery?}

MI is defined as the mental simulation of a given action, without actual execution. ${ }^{49}$ Imagery can be either 'internal' or 'external'. During 'internal' MI, subjects imagine themselves performing a given action from 'within'-that is, trying to mentally perceive the associated sensations and muscle contractions. This first person perspective relies on a kinaesthetic representation of the action, which involves the neural networks responsible for programming the actual actions. ${ }^{53}$ In contrast, 'external' or visual MI refers to self-visualisation of a movement from either the first person (ie, seeing the movement from one's own point of view) or the third person (ie, seeing oneself carrying out the task from outside) perspective. Third person MI engages neural areas involved in visual perception while specific cerebral structures are activated during internal versus external MI. ${ }^{53}$ Visual and kinaesthetic MIs are not mutually exclusive and may be experienced together, but an adapted training can promote the preferential use of one or the other perspective.

Cerebral correlates of motor imagery for upper limb movements Evidence from neuroimaging studies support the idea that the same neural structures are involved in actual execution and MI of a given motor act, ${ }^{4-51}$ both in healthy subjects and $\mathrm{PD}$ patients, ${ }^{52}$ although there is some debate regarding activation of the cerebral structures involved in motor execution, such as the primary motor or somatosensory cortices. ${ }^{49-5154}$ Nevertheless, in healthy subjects, the motor and premotor cortices, including the SMA, several parietal areas, the BG and cerebellum, are activated during MI, although to a lesser extent than during actual execution. ${ }^{49-51}$ In PD patients OFF medication compared with healthy controls, hypoactivation of frontal areas and the $\mathrm{BG}$, and hyperactivation of the premotor and parietal cortices and cerebellum have been reported. ${ }^{52}$

MI can also be used to study the neural networks involved in normal and pathological gait. However, to ensure good quality data, it is necessary to ascertain that subjects actually perform the MI task

\section{How to control the subject's engagement in motor imagery? Autonomic responses}

Several autonomic responses have been recorded during execution of MI tasks. Changes in cardiac and respiratory frequencies, electrodermal responses or muscular activity have been described, and appear to be related to the degree of imagined effort. ${ }^{55}$ However, these autonomic responses may have a large interindividual variability, and different responses may predominate in different subjects. In addition, to date, these controls have not been used to study gait. Behavioural controls appear easier to handle.

Table 1 Main cerebral activations reported during motor imagery of gait in healthy subjects and parkinsonian patients

\begin{tabular}{|c|c|c|c|}
\hline \multicolumn{4}{|l|}{ Motor imagery of natural gait } \\
\hline \multirow[b]{2}{*}{ Main activated brain areas } & \multirow[b]{2}{*}{ Techniques } & \multicolumn{2}{|l|}{ Studies } \\
\hline & & Healthy subjects & PD patients \\
\hline Frontal cortex (including DLPFC) & $\mathrm{H}_{2} \mathrm{O}^{15}$-PET, fMRI & $1561-697375$ & - \\
\hline Supplementary motor area (including both rostral and caudal SMA) & NIRS, SPECT, $\mathrm{H}_{2} 0^{15}$-PET, fMRI & $152660-75$ & 61 \\
\hline Lateral premotor cortex & $\mathrm{H}_{2} \mathrm{O}^{15}$-PET, fMRI & $15606263-727475$ & - \\
\hline Sensorimotor cortex & fMRI & 67 & - \\
\hline Cingulate cortex & $\mathrm{H}_{2} \mathrm{O}^{15}$-PET, fMRI & $1561-68707173-75$ & 61 \\
\hline Hippocampus & $\mathrm{H}_{2} \mathrm{O}^{15}$-PET, fMRI & 626771 & - \\
\hline Parahippocampal cortex & fMRI & $64-66 \quad 69-7173-75$ & - \\
\hline Fusiform cortex & $\mathrm{H}_{2} \mathrm{O}^{15}$-PET, fMRI & $6264-67707173$ & - \\
\hline Superior parietal lobule (including cuneus and precuneus) & $\mathrm{H}_{2} \mathrm{O}^{15}$-PET, fMRI & $60-717475$ & 61 \\
\hline Inferior parietal lobule (including supramarginal gyrus) & $\mathrm{H}_{2} \mathrm{O}^{15}$-PET, fMRI & $6367-697175$ & - \\
\hline Occipital cortex & $\mathrm{H}_{2} \mathrm{O}^{15}$-PET, fMRI & $6264-6668-717374$ & - \\
\hline Basal ganglia (caudate nucleus and/or putamen and/or subthalamic nucleus) & fMRI & $606164-6668707173-75$ & 61 \\
\hline Thalamus & fMRI & 68707175 & - \\
\hline Cerebellum & fMRI & $15606164-687173-75$ & 61 \\
\hline Midbrain (including cuneiform nucleus and PPN) & fMRI & $6164-667374$ & 61 \\
\hline Pons & fMRI & $64-6673$ & - \\
\hline
\end{tabular}


Table 2 Main brain activations reported during motor imagery of gait related tasks in healthy subjects and parkinsonian patients

\section{Other natural gait related imagery tasks}

\begin{tabular}{|c|c|c|c|}
\hline \multirow[b]{2}{*}{ Tasks (studies) } & \multirow[b]{2}{*}{ Techniques } & \multicolumn{2}{|l|}{ Main activated brain areas } \\
\hline & & Healthy subjects & PD patients \\
\hline Upright position ${ }^{63-66} 74$ & $\mathrm{H}_{2} \mathrm{O}^{15}-\mathrm{PET}, \mathrm{fMRI}$ & $\begin{array}{l}\text { FC (DLPFC), SMA, I-PMC, CC, HP, PHC, FfC, SPL (C, PrC), } \\
\text { IPL (SMG), TC, OC, BG (striatum), TL, CB, MB }\end{array}$ & - \\
\hline Gait initiation 637677 & $\mathrm{H}_{2} \mathrm{O}^{15}-\mathrm{PET}, \mathrm{fMRI}$ & FC (DLPFC), SMA, I-PMC, CC, SPL (PrC), IPL, OC & - \\
\hline Stepping/walking over an obstacle ${ }^{76} 77$ & fMRI & FC (DLPFC), SMA, I-PMC, CC, SPL (C, PrC), IPL, TC, OC, BG (putamen) & $\begin{array}{l}\text { CF, I-PMC, SMC, FfC, SPL } \\
(\mathrm{PrC}, \mathrm{C}), \mathrm{IPL}, \mathrm{TC}, \mathrm{OC}\end{array}$ \\
\hline Turn during walking ${ }^{71}$ & fMRI & FC, SMA, I-PMC, CC, HP, PHC, FfC, SPL (PrC), BG (putamen), TL, CB & - \\
\hline Gait termination ${ }^{76} 77$ & fMRI & FC (DLPFC), SMA, I-PMC, CC, SPL (PrC, C), IPL, TC, BG, TL & SPL $(\operatorname{PrC}, \mathrm{C}), \mathrm{TC}$ \\
\hline
\end{tabular}

Behavioural controls of motor imagery execution

Behavioural variables allow for indirect control of the actual performance of MI tasks. Chronometric characteristics of MI are similar to those of actual action. Several studies reported isochrony between actual and imagined action, ${ }^{55} 56$ especially for discrete and short duration movements involving the upper limbs. ${ }^{51} 5657$ The link between MI and cyclic motor skills appears more complex. ${ }^{57}$ Hence some studies reported overestimation while other observed underestimation of the time needed to actually perform a locomotion task. ${ }^{57}$ This is why studies of gait rest on another property of both actual and imagined movements-namely their complying with Fitts' law. ${ }^{58}$ According to this law, the time needed to perform a movement increases with its difficulty-that is, with the required amplitude and precision. To ensure that subjects actually perform the MI task, one can thus verify that its duration follows Fitts' law when the difficulty of the task is manipulated. ${ }^{58} 59$

\section{MOTOR IMAGERY OF LOCOMOTION IN CEREBRAL IMAGING: FROM HEALTHY SUBJECTS TO PD PATIENTS}

Having overcome the problems raised by the actual performance of complex movements, several teams have used MI coupled with fMRI or PET to decipher the cerebral substrates involved in the control of locomotion in healthy subjects. ${ }^{15} 26$ 60-77 Activations in the fronto-parietal (including SMA) and visuooccipital (including fusiform and parahippocampic gyri) cortices, BG and cerebellum have been reported during both MI of gait (table 1), and gait related tasks (table 2). These studies have also shown that a number of areas contribute to adjusting the locomotor behaviour to the environmental constraints ${ }^{63}$ or to the task complexity. ${ }^{69}$ Using fMRI, Jahn et al ${ }^{64}{ }^{65}$ reported activation in the dorsal pons during MI of standing and activation in the mesencephalic and cerebellar locomotor regions during MI of walking and running. In healthy subjects, Jahn et $a l^{66}$ also described lesser activation of the vestibular and somatosensory cortical areas during automated locomotion than during obstacle avoidance. Using MI of gait on a curved path, Wagner et $a l^{71}$ observed greater activation in the striatum contralateral to the turn, as well as parahippocampal and fusiform gyri activation. These two structures are involved in visually guided navigation. Comparing actual and imagined locomotion with PET and FMRI techniques, La Fougère et al ${ }^{73}$ reported activations in the primary motor and somatosensory cortices only during actual locomotion whereas the SMA and BG were activated during imagined locomotion. Moreover, the authors described greater activation within the pontomesencephalic tegmentum (including both the PPN and cuneiform nucleus) during imagined gait. Finally, Karachi et $a l^{15}$ reported activation of the PPN and cuneiform nucleus during imagery of fast gait when comparing imagining normal versus fast gait

These results were confirmed by fMRI studies in which the actual engagement of the participants in the MI task was controlled. ${ }^{59-6175}$ Figure 1 summarises the main results of these imaging studies.

To date, two studies have explored gait control in PD. ${ }^{61} 76$ Hypoactivation within the SMA and parietal lobule, as well as hyperactivation in the MLR, were seen during MI of gait in patients with FOG, compared with patients without FoG and matched controls. ${ }^{61}$

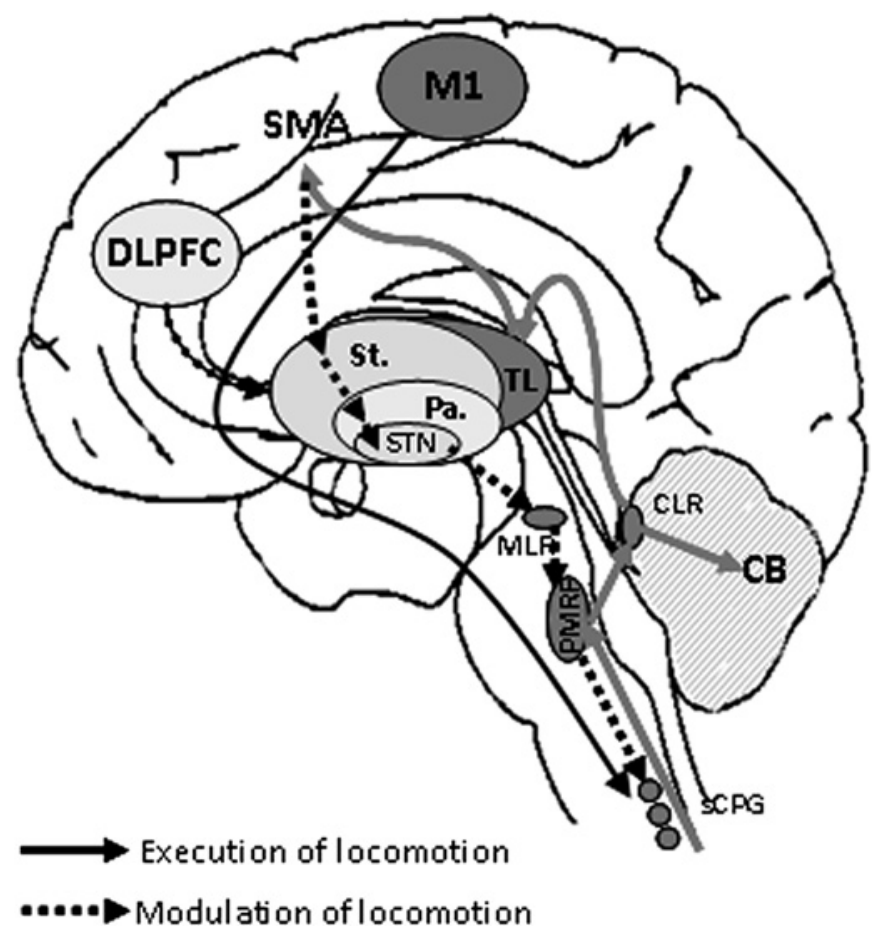

Figure 1 Schematic representation of the executive and planning networks of locomotion, illustrating hierarchical control from the cortex to the spinal cord. Solid arrows represent the ascending pathways; broken arrows represent the descending pathways. $\mathrm{CB}$, cerebellum; CLR, cerebellar locomotor region; DLPFC, dorsolateral prefrontal cortex; MLR, mesencephalic locomotor region; M1, primary motor cortex; $\mathrm{Pa}$, pallidum; PMRF, ponto-mesencephalic reticular formation; sCPG, spinal central pattern generator; SMA, supplementary motor area; St, striatum; STN, subthalamic nucleus; TL, thalamus. 
Figure 2 Pictures illustrating a motor imagery protocol using Fitts' law to control correct performance of the task. The protocol involves paths of various lengths and widths on which the subjects have to imagine walking and actually walk. Both imagined and actual walking times increase with path length and decrease with path width. A visual imagery task serves as an additional control. Subjects are asked to imagine a blue disk moving along the path. Visual imagery time is influenced by path length but not by path width (adapted from Bakker et $\left.a\right|^{59}$ ). (A) Short distance, wide path; $(B)$ long distance, narrow path; (C) long distance, wide path; (D) short distance, narrow path.
A

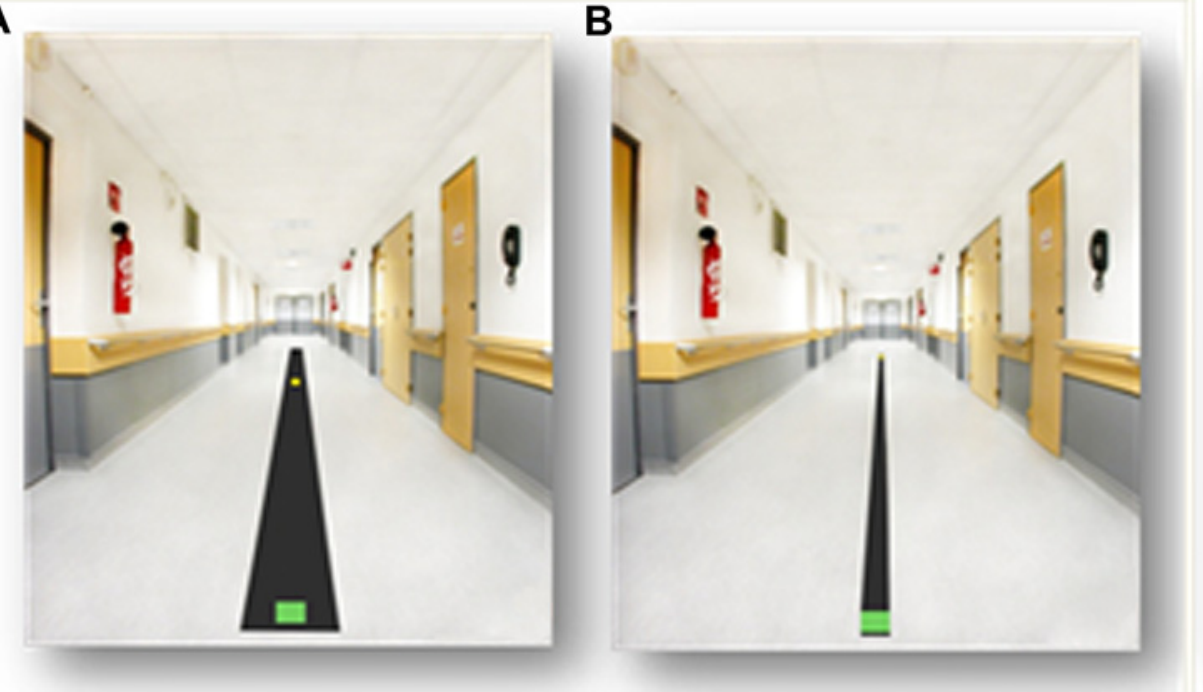

C

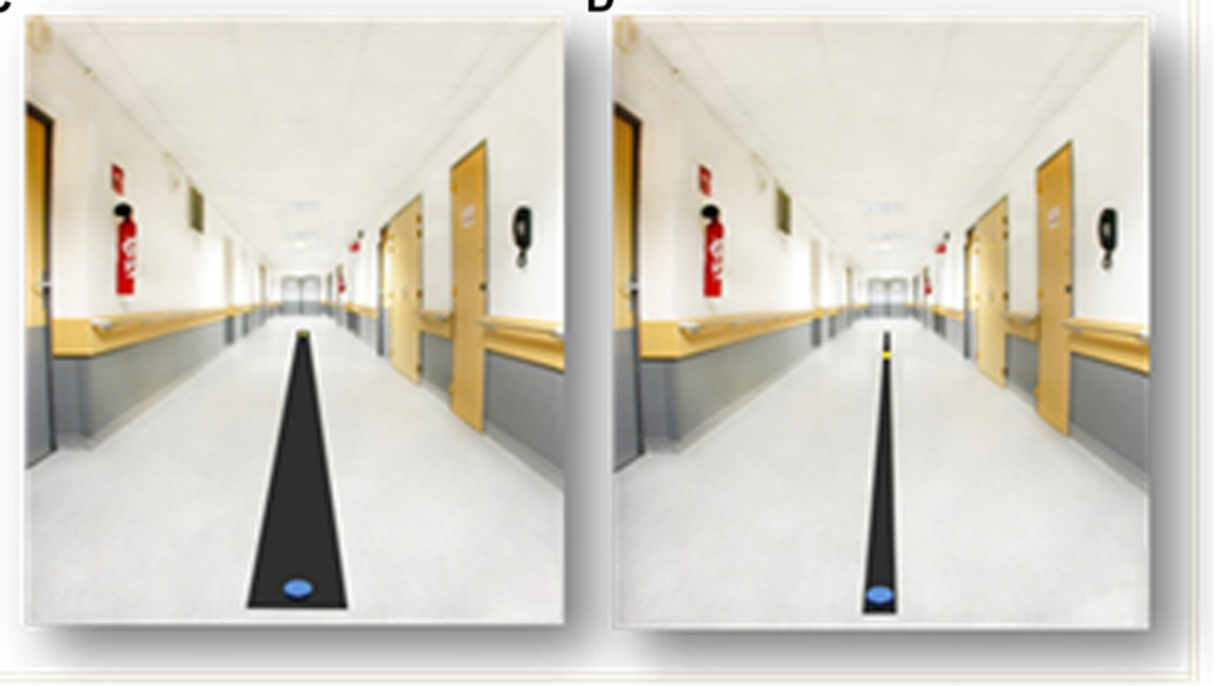

\section{Limitations of motor imagery}

These studies call for some comments. First, the MI perspective spontaneously used by the subjects is seldom controlled in spite of the differences in cerebral networks recruited during kinaesthetic and visual MI. In addition, the two perspectives do not engage motor areas in the same way, ${ }^{57} 72$ even if some common neural mechanisms exist. ${ }^{70}$ Second, all individuals do not have similar imagery skills and it thus appears necessary to assess both their MI ability and performance. The Movement Imagery Questionnaire $^{78}$ or the Kinaesthetic and Visual Imagery Questionnaire ${ }^{79}$-recently validated for $\mathrm{PD}$ patients ${ }^{80}$-enable one to measure the vividness of sensations perceived during visual and kinaesthetic MI and therefore the reliability of, say, their kinaesthetic imagery ability. Regarding gait, because it is an over learnt automated movement, it might be particularly difficult to voluntarily imagine. Yet, an adapted training focusing the subject's attention on the sensations triggered by gait execution can decrease visual processes and increase kinaesthetic ones, as demonstrated by Sacco et al. ${ }^{67}$

Some studies provided MI preliminary training ${ }^{15}$ 60-69 71-77 but did not control the correct performance of the MI tasks during image acquisition. Yet, to increase the reliability of MI of gait data, it appears necessary to make sure not only that participants use a kinaesthetic perspective and train them but also to monitor MI performance when the subjects are in the scanner, in order to control for proper task execution. Different methods, based mainly on the temporal similarities of actual and imagined movements, can be used to ascertain that participants actually imagine the target movement during brain image acquisition. The Time Dependant Motor Imagery screening test consists in recording the number of movements imagined over a defined period of time, following the assumption that the number of imagined movements will increase with time. The Temporal Congruence Test involves recording the duration of actual and represented movements to evaluate their chronometric concordance. Bakker et al $l^{60}$ used Fitts' law, asking healthy subjects to imagine walking along paths of various lengths and widths, and then to actually walk along the same paths (figure 2). The results confirmed that actual and imagined walking times were similarly affected by the changes in difficulty of the task. Bakker et al also used a visual imagery task, during which subjects were to imagine seeing a small disk moving along the path. The authors reported that, unlike imagined walking times, the visual imagery times increased with path length, but were less sensitive to its width. ${ }^{60}$

Finally, one might question the use of motor imagery (MI) protocols which require high level cognitive processing in patients with gait disorders. Indeed, the link between frontal 
lobe dysfunction and gait disorders is clearly established. ${ }^{81} 82$ However, although patients with executive function impairments often display gait impairments and falls, a number of PD patients with gait disorders and FOG have well preserved executive functioning. ${ }^{80}$ In addition, providing specific kinaesthetic MI training to patients before data acquisition both increases the quality of these processes in the selected individuals and contributes to the detection and exclusion of patients whose level of executive functioning can no longer support reliable MI.

\section{CONCLUSION}

Complementary brain imaging techniques can enable one to identify the neural correlates of normal and $\mathrm{PD}$ gait. The consistency of published results strongly suggests that MI is a well suited method for exploring the neural networks of gait. However, the methodology can still be improved by including patients presenting with clearly defined gait symptoms on the one hand, and by providing kinaesthetic training on the other. Such precautions might not only lead to better understanding of the links between the cortical, subcortical and brainstem areas involved in different forms of gait disorders, but also clarify the effects of antiparkinsonian treatments and DBS on these disabling symptoms.

Contributors AM performed the systematic literature search, wrote the first draft of the paper as well as drafts of the revised papers. PP and BD supervised the writing of this review paper and critically reviewed the draft.

Funding This work was supported by the Centre Hospitalier Universitaire de Grenoble, grant No PHRC 2009, and the University Joseph Fourier. AM also thanks the France Parkinson Association for her financial support.

Competing interests None.

Provenance and peer review Not commissioned; externally peer reviewed.

\section{REFERENCES}

1. Bloem BR, Hausdorff JM, Visser JE, et al. Falls and freezing of gait in Parkinson's disease: a review of two interconnected, episodic phenomena. Mov Disord 2004;19:871-84.

2. Giladi N, Kao R, Fahn S. Freezing phenomenon in patients with parkinsonian syndromes. Mov Disord 1997:12:302-5.

3. Factor SA. The clinical spectrum of freezing of gait in atypical parkinsonism. Mov Disord 2008:23(Suppl 2):431-8.

4. Achiron A, Ziv I, Goren M, et al. Primary progressive freezing gait. Mov Disord 1993; 8:293-7.

5. Ferraye MU, Debû B, Fraix V, et al. Effects of subthalamic nucleus stimulation and levodopa on freezing of gait in Parkinson disease. Neurology 2008;70:1431-7.

6. Grillner S, Hellgren J, Menard A, et al. Mechanisms for selection of basic motor programs - roles for the striatum and pallidum. Trends Neurosci 2005;28:364-70.

7. Takakusaki K, Tomita N, Yano M. Substrates for normal gait and pathophysiology of gait disturbances with respect to the basal ganglia dysfunction. J Neurol 2008:255 (Suppl 4):19-29.

8. Bonnet AM, Loria Y, Saint-Hilaire MH, et al. Does long-term aggravation of Parkinson's disease result from nondopaminergic lesions? Neurology 1987; 37:1539-42

9. Pahapill PA, Lozano AM. The pedunculopontine nucleus and Parkinson's disease. Brain 2000:123:1767-83

10. Tohgi H, Abe T, Takahashi S. The effects of I-threo-3,4-dihydrox dihydroxyphenylserine on the total norepinephrine and dopamine concentrations in the cerebrospinal fluid and freezing gait in parkinsonian patients. J Neural Transm Park Dis Dement Sect 1993:5:27-34.

11. Zarow C, Lyness SA, Mortimer JA, et al. Neuronal loss is greater in the locus coeruleus than nucleus basalis and substancia nigra in Alzheimer and Parkinson diseases. Arch Neurol 2003;60:337-41.

12. Rommelfanger KS, Edwards GL, Freeman KG, et al. Norepinephrine loss produces more profound motor deficits than MPTP treatment in mice. Proc Natl Acad Sci U S A 2007; 104:13804-9.

13. Devos D, Defebvre L, Bordet R. Dopaminergic and non-dopaminergic pharmacological hypotheses for gait disorders in Parkinson's disease. Fundam Clin Pharmacol 2010;24:407-21.

14. Jenkinson N, Nandi D, Miall RC, et al. Pedunculopontine nucleus stimulation improves akinesia in a Parkinsonian monkey. Neuroreport 2004;15:2621-4.

15. Karachi C, Grabli D, Bernard FA et al. Cholinergic mesencephalic neurons are involved in gait and postural disorders in Parkinson disease. J Clin Invest 2010;120:2745-54.
16. Kuo SH, Kenney C, Jankovic J. Bilateral pedunculopontine nuclei strokes presenting as freezing of gait. Mov Disord 2008;23:616-19.

17. Hirsch EC, Graybiel AM, Duyckaerts $C$, et al. Neuronal loss in the pedunculopontine tegmental nucleus in Parkinson disease and in progressive supranuclear palsy. Proc Natl Acad Sci U S A 1987;84:5976-80.

18. Chastan N, Do MC, Bonneville F, et al. Gait and balance disorders in Parkinson's disease: impaired active braking of the fall of centre of gravity. Mov Disord 2009:24:188-95

19. Lee PH, Yong SW, Ahn YH, et al. Correlation of midbrain diameter and gait disturbance in patients with idiopathic normal pressure hydrocephalus. J Neurol 2005;252:958-63.

20. Stefani A, Lozano AM, Peppe A, et al. Bilateral deep brain stimulation of the pedunculopontine and subthalamic nuclei in severe Parkinson's disease. Brain 2007; 130:1596-607.

21. Plaha $\mathbf{P}$, Gill SS. Bilateral deep brain stimulation of the pedunculopontine nucleus for Parkinson's disease. Neuroreport 2005;16:1883-7.

22. Ferraye MU, Debû $B$, Fraix $\mathrm{V}$, et al. Effects of pedunculopontine nucleus area stimulation on gait disorders in Parkinson's disease. Brain 2010:133:205-14.

23. Moro E, Hamani C, Poon YY, et al. Unilateral pedunculopontine stimulation improves falls in Parkinson's disease. Brain 2010:133:215-24.

24. do Nascimento OF, Nielsen KD, Voigt M. Influence of directional orientations during gait initiation and stepping on movement-related cortical potentials. Behav Brain Res 2005:161:141-54.

25. Gwin JT, Gramann K, Makeig S. Electrocortical activity is coupled to gait cycle phase during treadmill walking. Neuroimage 2011;54:1289-96.

26. Miyai I, Tanabe HC, Sase I, et al. Cortical mapping of gait in humans: an near infrared spectroscopic topography study. Neuroimage 2001;14:1186-92.

27. Sahyoun C, Floyer-Lea A, Johansen-Berg H, et al. Towards an understanding of gait control: brain activation during the anticipation, preparation, and execution of foot movements. Neuroimage 2004;21:568-75.

28. Fabre N, Brefel C, Sabatini U. Striatal dopa and glucose metabolism in PD patients with freezing of gait. Mov Disord 1998;13:677-83.

29. Matsui H, Udaka F, Miyoshi T, et al. Three dimensional stereotaxic surface projection study of freezing of gait and brain perfusion image in Parkinson's disease. Mov Disord 2005;20:1272-7.

30. Bartels AL, de Jong BM, Giladi N, et al. Striatal dopa and glucose metabolism in PD patients with freezing of gait. Mov Disord 2006;21:1326-32.

31. Mito Y, Yoshida K, Yabe I, et al. Brain SPECT analysis by 3D-SSP and phenotype of Parkinson's disease. J Neurol Sci 2006;241:67-72.

32. Bohnen NI, Muller ML, Koeppe RA, et al. History of falls in Parkinson's disease is associated with reduced cholinergic activity. Neurology 2009;73:1670-6.

33. Bosman T, Van Laere K, Santens P. Anatomically standardized ${ }^{99 m} \mathrm{TC}-\mathrm{ECD}$ brain perfusion SPECT allows accurate differentiation between healthy volunteers, multiple system atrophy and idiopathic Parkinson's disease. Eur J Nucl Med 2003;30:16-24.

34. Eckert T, Barnes A, Dhawan V, et al. FDG PET in the differential diagnosis of parkinsonian disorders. Neuroimage 2005;26:912-21.

35. Park HK, Kim JS, Im KC, et al. Functional brain imaging in pure akinesia with gait freezing: [18F] FDG PET and [18F] FP-CIT PET analyses. Mov Disord 2009;24:237-45

36. Ouchi $\mathrm{Y}$, Kanno $\mathrm{T}$, Okada $\mathrm{H}$, et al. Changes in dopamine availability in the nigrostriatal and mesocortical dopaminergic systems by gait in Parkinson's disease. Brain 2001;124:784-92.

37. del Olmo MF, Arias P, Furio MC, et al. Evaluation of the effect of training using auditory stimulation on rhythmic movements in parkinsonian patients-a combined motor and [18F]-FDG PET study. Parkinsonism Relat Disord 2006;12:155-64.

38. Suzuki M, Miyai I, Ono T, et al. Prefrontal and premotor cortices are involved in adapting walking and running speed on the treadmill: an optical imaging study. Neuroimage 2004:23:1020-6.

39. Holtzer R, Mahoney JR, Izzetoglu M, et al. fNIRS study of walking and walking while talking in young and old individuals. J Gerontol A Biol Sci Med Sci 2011;66:879-87.

40. Fukuyama $\mathbf{H}$, Ouchi $\mathrm{Y}$, Matsuzaki $\mathrm{S}$, et al. Brain functional activity during gait in normal subjects: a SPECT study. Neurosci Lett 1997:228:183-6.

41. Hanakawa T, Katsumi $Y$, Fukuyama $\mathrm{H}$, et al. Mechanisms underlying gait disturbances in Parkinson's disease: a single photon emission computed tomography study. Brain 1999;122:1271-82.

42. Hanakawa $\mathrm{T}$, Fukuyama $\mathrm{H}$, Katsumi $\mathrm{Y}$, et al. Enhanced lateral premotor activity during paradoxical gait in Parkinson's disease. Ann Neurol 1999;45:329-36.

43. Iseki K, Hanakawa T, Hashikawa K et al. Gait disturbances associated with white matter changes: a gait analysis and blood flow study. Neuroimage 2010;49:1659-66.

44. Lyoo CH, Aalto S, Rinne J0, et al. Different cerebral cortical areas influence the effect of subthalamic nucleus stimulation on parkinsonian motor deficits and freezing of gait. Mov Disord 2007:22:2176-82.

45. Strafella AP, Lozano AM, Ballanger B, et al. rCBF changes associated with PPN stimulation in a patient with Parkinson's disease: a PET study. Mov Disord 2008;23:1051-4

46. Ballanger B, Lozano AM, Moro E, et al. Cerebral blood flow changes induced by pedonculopontine nucleus stimulation in patients with advanced Parkinson's disease: a $\left[{ }^{15} \mathrm{O}\right] \mathrm{H}_{2} \mathrm{O}$ PET study. Hum Brain Mapp 2009:30:3901-9.

47. Khan S, Gill SS, Mooney L, et al. Combined pedunculopontine-subthalamic stimulation in Parkinson's disease. Neurology 2012;78:1090-5. 
48. Malouin F, Richards CL. Mental practice for relearning locomotor skills. Phys Ther 2010;90:240-51

49. Jeannerod M. The representing brain: neural correlates of motor intention and imagery. Behav Brain Sci 1994;17:187-245.

50. Jeannerod M. Mental imagery in a motor context. Neuropsychologia 1995; 33:1419-32.

51. Decety J. The neurophysiological basis of motor imagery. Behav Brain Res 1996; 77:45-52

52. Thobois $\mathbf{S}$, Dominey PF, Decety $\mathrm{J}$, et al. Motor imagery in normal subjects and in asymmetrical Parkinson's disease: a PET study. Neurology 2000;55:996-1002.

53. Guillot A, Collet $\mathrm{C}$, Nguyen VA, et al. Brain activity during visual versus kinesthetic imagery: an fMRI study. Hum Brain Mapp 2009;30:2157-72.

54. Nair DG, Purcott KL, Fuchs A, et al. Cortical and cerebellar activity of the human brain during imagined and executed unimanual and bimanual action sequences: a functional MRI study. Brain Res Cogn Brain Res 2003:15:250-60.

55. Decety J, Jeannerod M, Durozard D, et al. Central activation of autonomic efforts during mental simulation of motor actions in man. J Physiol 1993;461:549-63.

56. Decety J, Michel F. Comparative analysis of actual and mental movement times in two graphic tasks. Brain Cogn 1989;11:87-97.

57. Guillot A, Collet C. Duration of mentally simulated movement: a review. J Mot Behav 2005:37:10-19.

58. Fitts PM. The informed capacity of the human motor system in controlling the amplitude of movements. J Exp Psychol 1954;47:381-91.

59. Bakker M, De Lange FP, Stevens JA, et al. Motor imagery of gait: a quantitative approach. Exp Brain Res 2007:179:497-504.

60. Bakker M, De Lange FP, Helmich RC, et al. Cerebral correlates of motor imagery of normal and precision gait. Neuroimage 2008;41:998-1010.

61. Snijders AH, Leunissen I, Bakker M, et al. Gait-related cerebral activations in patients with Parkinson's disease with freezing of gait. Brain 2011;134:59-72.

62. Ghaem $\mathbf{0}$, Mellet $\mathrm{E}$, Crivello $\mathrm{F}$, et al. Mental navigation along memorized routes activates the hippocampus, precuneus and insula. Neuroreport 1997;10: 739-44.

63. Malouin F, Richards CL, Jackson PL, et al. Brain activations during motor imagery of locomotor-related tasks: a PET study. Hum Brain Mapp 2003;19:47-62.

64. Jahn K, Deutschländer A, Stephan T, et al. Brain activation patterns during imagined stance and locomotion in functional magnetic resonance imaging. Neuroimage 2004;22:1722-31.

65. Jahn K, Deutschländer A, Stephan T, et al. Imaging human supraspinal locomotor centers in brainstem and cerebellum. Neuroimage 2008;39:786-92.
66. Jahn K, Deutschlander A, Stephan T, et al. Supraspinal locomotor control in quadrupeds and humans. Prog Brain Res 2008;171:353-61.

67. Sacco K, Cauda F, Cerliani $L$, et al. Motor imagery of walking following training in locomotor attention: the effects of 'the tango lesson'. Neuroimage 2006;32:1441-9.

68. Wang C, Wai Y, Weng Y, et al. The cortical modulation from the external cues during gait observation and imagination. Neurosci Lett 2008:443:232-5.

69. Wang C, Wai Y, Kuo B, et al. Cortical control of gait in healthy humans: an fMRI study. J Neural Transm 2008;115:1149-58.

70. Iseki K, Hanakawa T, Shinozaki J, et al. Neural mechanisms involved in mental imagery and observation of gait. Neuroimage 2008;41:1021-31.

71. Wagner J, Stephan T, Kalla R, et al. Mind the bend: cerebral activations associated with mental imagery of walking along a curved path. Exp Brain Res 2008;191:247-55.

72. Flanagin VL, Wutte M, Glasauer $\mathrm{S}$, et al. Cortical activations during imagined passive and active whole body movement. Ann NY Acad Sci 2009:1164:372-5.

73. La Fougère $\mathbf{C}$, Zwergal $A$, Rominger $A$, et al. Real versus imagined locomotion: a [18F]-FDG PET-fMRI comparison. Neuroimage 2010:50:1589-98.

74. Zwergal A, Linn J, Xiong G, et al. Aging of human supraspinal locomotor and postural control in fMRI. Neurobiol Aging 2010;33:1073-84.

75. Cremers J, Dessoullières A, Garraux G. Hemispheric specialization during mental motor imagery of brisk walking. Hum Brain Mapp 2011;33:873-82.

76. Wai YY, Wang JJ, Weng YH, et al. Cortical involvement in a gait-related imagerytask: comparison between Parkinson's disease and normal aging. Parkinsonism Relat Disord 2012;18:537-42..

77. Wang J, Wai $Y$, Weng $Y$, et al. Functional MRI in the assessment of cortical activation during gait-related imaginery tasks. J Neural Trans 2009:116:1087-92.

78. Hall C, Pongrac J. Movement Imagery Questionnaire. London: University of Western Ontario, 1983

79. Malouin F, Richards CL, Jackson PL, et al. The Kinaesthetic and Visual Imagery Questionnaire (KVIO) for assessing motor imagery in persons with physical disabilities: a reliability and construct validity study. J Neurol Phys Ther 2007:31:20-9.

80. Randhawa B, Harris S, Boyd LA. The kinaesthetic and visual imagery questionnaire is a reliable tool for individuals with Parkinson's disease. J Neurol Phys Ther 2010;34:161-7.

81. Amboni M, Cozzolino $\mathrm{A}$, Longo $\mathrm{K}$, et al. Freezing of gait and executive functions in patients with Parkinson's disease. Mov Disord 2008:23:395-400.

82. Giladi N. Gait and mental function: the interplay between walking, behavior and cognition. J Neural Transm 2007:114:1241-2. 